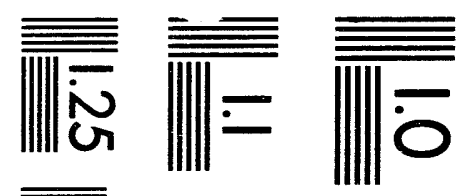

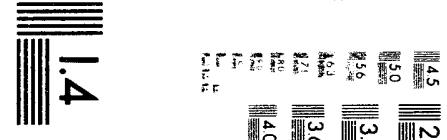

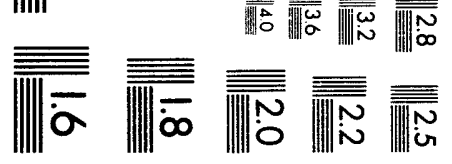



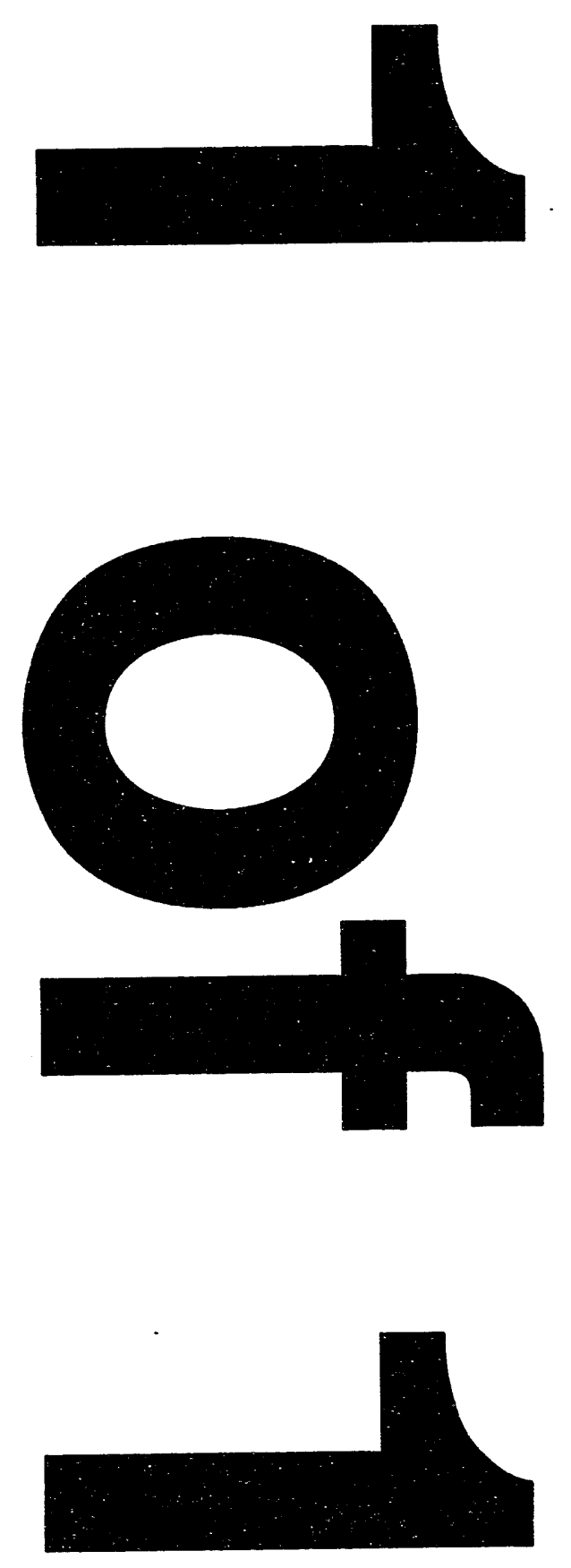


\title{
Numerical Procedure for Planetary Wave Solution
}

\author{
W. K. Choi
}

D. J. Wuebbles

\section{September 1993}

This is an informal report intended primarily for internal or limited external distribution. The opinions and conclusions stated are those of the author and may or may not be those of the Laboratory.

Work performed under the auspices of the U.S. Department of Energy by the Lawrence Livermore National Laboratory under Contract W-7405-Eng-48. 


\section{DISCLAIMER}

This document was prepared as an account of work sponsored by an agency of the United States Government. Neither the United States Government nor the University of California nor any of their employees, makes any warranty, express or implied, or assumes any legal liability or responsibility for the accuracy, completeness, or usefulness of any information, apparatus, product, or process disclosed, or represents that its use would not infringe privately own rights. Reference herein to any specific commercial products, process, or service by trade name, trademark, manufacturer, or otherwise, does not necessarily constitute or imply its endorsement, recommendation, or favoring by the United States Government or the University of California. The views and opinions of authors expressed herein do not necessarily state or reflect those of the United States Government or the University of California, and shall not be used for advertising or product endorsement purposes.

This report has been reproduced directly from the best available copy.

Available to DOE and DOE contractors from the Office of Scientific and Technical Information P.O. Box 62, Oak Ridge, TN 37831

Prices available from (615) 576-8401, FTS 626-8401

Available to the public from the National Teciunical Information Service

U.S. Department of Commerce 5285 Port Royal Rd.

Springfield, VA 22161 


\title{
Numerical Procedure for Planetary Wave Solution
}

\author{
Woo Kap Choi and Donald J. Wuebbles \\ Global Climate Research Division \\ Lawrence Livermore National Laboratory
}

\section{Introduction}

The newly-developed LLNL two-dimensional chemical-radiative-transport model requires a knowledge of the EP flux divergence as an input momentum forcing. The major contributions for this forcing term come from the synoptic wave in the troposphere, the planetary wave in the stratosphere and mesosphere and the gravity wave in the upper mesosphere.

The major source of the zonal momentum forcing in the middle atmosphere is the nonlinear planetary wave breaking. This planetary wave breaking also plays a significant role of mixing the chemical tracers. Garcia (1991) suggested a way of parameterizing the planetary wave breaking by using linear damping of the primary wave. In this note we describe the procedure of obtaining the wave solution for the parameterization.

\section{Formulation for the propagation of the stationary planetary wave}

In the spherical and the log-pressure coordinate system, the coordinates are defined by $d x=a \cos \phi d \lambda, d y=a d \phi$ and $d z=-H d \ln p$. 
The perturbation potential vorticity equation with the Rayleigh friction and the Newtonian cooling is written as (Matsuno, 1970; Holton, 1975)

$$
\left(\frac{\partial}{\partial t}+\bar{u} \frac{\partial}{\partial x}\right) q_{(M)}^{\prime}+\frac{1}{f} \frac{\partial \Phi^{\prime}}{\partial x} \frac{\partial \bar{q}}{\partial y}=-\gamma\left(\zeta_{g}-\frac{1}{f^{2}} \frac{d f}{d y} \frac{\partial \Phi^{\prime}}{\partial y}\right)-\frac{f}{\rho_{0}} \frac{\partial}{\partial z}\left(\frac{\rho_{0}}{N^{2}} \alpha \frac{\partial \Phi^{\prime}}{\partial z}\right)
$$

where $\Phi^{\prime}$ is perturbation geopotential, $\gamma$ is the Rayleigh friction coefficient, $\alpha$ is the Newtonian cooling coefficient and $\zeta_{g}$ is the geostrophic vorticity.

The potential vorticity $q_{(M)}^{\prime}$ is defined by

$$
q_{(M)}^{\prime}=\frac{1}{f} \frac{\partial^{2} \Phi^{\prime}}{\partial x^{2}}+\frac{f}{\cos \phi} \frac{\partial}{\partial y}\left(\frac{\cos \phi}{f^{2}} \frac{\partial \Phi^{\prime}}{\partial y}\right)+\frac{f}{\rho_{0}} \frac{\partial}{\partial z}\left(\frac{\rho_{0}}{N^{2}} \frac{\partial \Phi^{\prime}}{\partial z}\right)
$$

and the gradient of the zonal mean potential vorticity is

$$
\frac{\partial \bar{q}}{\partial y}=\frac{2 \Omega \cos \phi}{a}-\frac{\partial}{\partial y}\left[\frac{1}{\cos \phi} \frac{\partial}{\partial y}(\bar{u} \cos \phi)\right]-\frac{f^{2}}{\rho_{0}} \frac{\partial}{\partial z}\left(\frac{\rho_{0}}{N^{2}} \frac{\partial \bar{u}}{\partial z}\right)
$$

where $\rho_{0}$ is the global mean density and $N^{2}$ is the buoyancy frequency squared defined by

$$
N^{2}=\frac{R}{H}\left(\frac{d T_{0}}{d z}+\frac{\kappa T_{0}}{H}\right)
$$

where $T_{0}$ is the global mean temperature and function of height only.

The bottom boundary condition (Andrews et al., 1987) can be written through the thermodynamic energy equation as

$\left(\frac{\partial}{\partial t}+\bar{u} \frac{\partial}{\partial x}\right)\left(\frac{\partial \Phi^{\prime}}{\partial z}-\frac{N^{2} H}{R T_{0}} \Phi^{\prime}\right)-\left(\frac{\partial \bar{u}}{\partial z}-\frac{N^{2} H}{R T_{0}} \bar{u}\right) \frac{\partial \Phi^{\prime}}{\partial x}=-\alpha \frac{\partial \Phi^{\prime}}{\partial z}-\frac{N^{2} H}{R T_{0}} g W^{\prime}$ at $z=0$

where $W^{\prime}$ is the geometric vertical velocity.

The perturbation geopotential is next written as 


$$
\Phi^{\prime}=e^{z / 2 H} \operatorname{Re}\left\{\Psi(y, z, t) e^{i k x}\right\}
$$

and then the potential vorticity equation divided by $e^{i k x} e^{z / 2 H}$ becomes

$$
\begin{aligned}
\left(\frac{\partial}{\partial t}\right. & +i k \bar{u}+\gamma) \frac{q_{(M)}^{\prime}}{e^{i k x} e^{2 / 2 H}}+\frac{i k}{f} \frac{\partial \bar{q}}{\partial y} \Psi \\
& =-\frac{f}{N^{2}} \alpha^{\prime}\left[\frac{\partial^{2} \Psi}{\partial z^{2}}+N^{2} \frac{\partial N^{-2}}{\partial z} \frac{\partial \Psi}{\partial z}+\frac{1}{2 H}\left(N^{2} \frac{\partial N^{-2}}{\partial z}-\frac{1}{2 H}\right) \Psi\right]-\frac{f}{N^{2}} \frac{\partial \alpha}{\partial z}\left(\frac{\partial \Psi}{\partial z}+\frac{\Psi}{2 H}\right)
\end{aligned}
$$

where $\alpha^{\prime}=\alpha-\gamma$ and

$$
\begin{aligned}
\frac{q_{(M)}^{\prime}}{e^{i k x} e^{z / 2 H}}=-\frac{k^{2}}{f} \Psi & +\frac{f}{\cos \phi} \frac{\partial}{\partial y}\left(\frac{\cos \phi}{f^{2}} \frac{\partial \Psi}{\partial y}\right) \\
+ & \frac{f}{N^{2}}\left[\frac{\partial^{2} \Psi}{\partial z^{2}}+N^{2} \frac{\partial N^{-2}}{\partial z} \frac{\partial \Psi}{\partial z}+\frac{1}{2 H}\left(N^{2} \frac{\partial N^{-2}}{\partial z}-\frac{1}{2 H}\right) \Psi\right]
\end{aligned}
$$

The boundary condition is similarly written in terms of $\Psi$ as

$$
\begin{aligned}
\left(\frac{\partial}{\partial t}+i k \bar{u}\right)\left[\frac{\partial \Psi}{\partial z}+\left(\frac{1}{2 H}-\frac{N^{2} H}{R T_{0}}\right) \Psi\right]-i k\left(\frac{\partial \bar{u}}{\partial z}-\frac{N^{2} H}{R T_{0}} \bar{u}\right) \Psi & \\
& =-\alpha\left(\frac{\partial \Psi}{\partial z}+\frac{\Psi}{2 H}\right)-\frac{N^{2} H}{R T_{0}} \frac{g W^{\prime}}{e^{i k x}}
\end{aligned}
$$

Neglecting the temporal variation and multiplying the equation (7) by $f /(i k \bar{u}+\gamma)$ gives

$$
A \frac{\partial^{2} \Psi}{\partial y^{2}}+B \frac{\partial^{2} \Psi}{\partial z^{2}}+C \frac{\partial \Psi}{\partial y}+D \frac{\partial \Psi}{\partial z}+E \Psi=0
$$

where

$$
\begin{aligned}
& A=1 \\
& B=\frac{f^{2}}{N^{2}}\left(1+\frac{\alpha^{\prime}}{i k \bar{u}+\gamma}\right)
\end{aligned}
$$




$$
\begin{aligned}
& C=-\frac{\tan \phi}{a}-\frac{2}{a \tan \phi} \\
& D=\frac{f^{2}}{N^{2}}\left[N^{2} \frac{\partial N^{-2}}{\partial z}+\frac{1}{i k \bar{u}+\gamma}\left(\alpha^{\prime} N^{2} \frac{\partial N^{-2}}{\partial z}+\frac{\partial \alpha}{\partial z}\right)\right] \\
& E=n_{k}^{2}+\frac{1}{i k \bar{u}+\gamma} \frac{f^{2}}{2 H N^{2}}\left[\alpha^{\prime}\left(N^{2} \frac{\partial N^{-2}}{\partial z}-\frac{1}{2 H}\right)+\frac{\partial \alpha}{\partial z}\right]
\end{aligned}
$$

where

$$
n_{k}^{2}=\frac{1}{\bar{u}-i \gamma / k} \frac{\partial \bar{q}}{\partial y}-k^{2}+\frac{f^{2}}{2 H N^{2}}\left(N^{2} \frac{\partial N^{-2}}{\partial z}-\frac{1}{2 H}\right)
$$

is the square of the refractive index for zonal wave number $k$. Above relations show that the singularities are removed by introducing the Raleigh friction coefficient.

The boundary condition can be written similarly. Before doing that, it is better to write the geometric vertical velocity as

$$
W^{\prime}=\bar{u}_{s} \frac{\partial h}{\partial x}
$$

where the surface height is $h=\operatorname{Re}\left\{\bar{h}(y) e^{i k x}\right\}$. The surface wind $\bar{u}_{s}$ may be prescribed and thus may be different from the $\bar{u}(z=0)$. The vertical velocity is thus written as

$$
W^{\prime}=i k \bar{u}_{s} \bar{h} e^{i k x}
$$

For the steady wave, the bottom boundary condition divided by $i k e^{i k x}$ then can be written using (13) as

$$
X \frac{\partial \Psi}{\partial z}+Y \Psi=Z
$$

where 


$$
\begin{aligned}
& X=\bar{u}+\frac{\alpha}{i k} \\
& Y=\frac{1}{2 H}\left(\bar{u}+\frac{\alpha}{i k}\right)-\frac{\partial \bar{u}}{\partial z} \\
& Z=-\frac{N^{2} H}{R T_{0}} g \bar{h} \bar{u}_{s}
\end{aligned}
$$

The numerical scheme for solving (10) and (14) is based on Lindzen and Kuo (1969). It is described in the appendix in detail. Once wave solutions are obtained, we can proceed by following Garcia (1991). 


\section{APPENDIX}

\section{Numerical Solution Procedure}

We consider a second order elliptic partial differential equation for $\psi$ written

as

$$
a \frac{\partial^{2} \psi}{\partial y^{2}}+b \frac{\partial^{2} \psi}{\partial y \partial z}+c \frac{\partial^{2} \psi}{\partial z^{2}}+d \frac{\partial \psi}{\partial y}+e \frac{\partial \psi}{\partial z}+f \psi=g
$$

and the bottom and top boundary conditions are

$$
\begin{array}{ll}
p \frac{\partial \psi}{\partial y}+q \frac{\partial \psi}{\partial z}+r \psi=s & \text { at the bottom } \\
t \frac{\partial \psi}{\partial y}+u \frac{\partial \psi}{\partial z}+v \psi=h & \text { at the top }
\end{array}
$$

The domain of the problem is from the South pole to the North pole latitudinally and from the surface of the earth to the middle atmosphere verticaliy. The function $\psi$ is zero at the both poles. The meridional index $j$ changes from 0 at the South Pole to $M+1$ at the North Pole latitudinally and the vertical index $k$ changes from 1 at the surface to $N+1$ at the top of the domain vertically. The equation is represented in the finite difference form at an interior point $(j$ is from 1 to $M ; k$ is from 2 to $N$ ) as

$$
\begin{aligned}
& a_{j, k} \frac{\psi_{j+1, k}-2 \psi_{j, k}+\psi_{j-1, k}}{\Delta y^{2}}+\frac{b_{j, k}}{2 \Delta z}\left(\frac{\psi_{j+1, k+1}-\psi_{j-1, k+1}}{2 \Delta y}-\frac{\psi_{j+1, k-1}-\psi_{j-1, k-1}}{2 \Delta y}\right) \\
& +c_{j, k} \frac{\psi_{j, k+1}-2 \psi_{j, k}+\psi_{j, k-1}}{\Delta y^{2}}+d_{j, k} \frac{\psi_{j+1, k}-\psi_{j-1, k}}{2 \Delta y}+e_{j, k} \frac{\psi_{j, k+1}-\psi_{j, k-1}}{2 \Delta z}+f_{j, k} \psi_{j, k}=g_{j, k}
\end{aligned}
$$

where $\Delta y$ and $\Delta z$ are intervals in the meridional and vertical directions respectively. Rearranging the terms yields 


$$
\begin{aligned}
\frac{b_{j, k}}{4 \Delta y \Delta z} \psi_{j-1, k-1}+\left(\frac{c_{j, k}}{\Delta z^{2}}-\frac{e_{j, k}}{2 \Delta z}\right) \psi_{j, k-1}-\frac{b_{j, k}}{4 \Delta y \Delta z} \psi_{j+1, k-1} \\
+\left(\frac{a_{j, k}}{\Delta y^{2}}-\frac{d_{j, k}}{2 \Delta y}\right) \psi_{j-1, k}+\left(f_{j, k}-\frac{2 a_{j, k}}{\Delta y^{2}}-\frac{2 c_{j, k}}{\Delta z^{2}}\right) \psi_{j, k}+\left(\frac{a_{j, k}}{\Delta y^{2}}+\frac{d_{j, k}}{2 \Delta y}\right) \psi_{j+1, k} \\
\quad-\frac{b_{j, k}}{4 \Delta y \Delta z} \psi_{j-1, k+1}+\left(\frac{c_{j, k}}{\Delta z^{2}}+\frac{e_{j, k}}{2 \Delta z}\right) \psi_{j, k+1}+\frac{b_{j, k}}{4 \Delta y \Delta z} \psi_{j+1, k+1}=g_{j, k}
\end{aligned}
$$

The finite difference forms written at all the interior points consists of $M \times(N-1)$ equations and $M \times(N+1)$ unknowns since $\psi_{0, k}=\psi_{M+1, k}=0$. (A5) can be written as a matrix form

$$
\mathbf{A}_{k} \boldsymbol{\Psi}_{k-1}+\mathbf{B}_{k} \boldsymbol{\Psi}_{k}+\mathbf{C}_{k} \boldsymbol{\Psi}_{k+1}=\mathbf{g}_{k}, \quad k=2, \cdots, N
$$

where the tridiagonal coefficient matrix $\mathbf{A}_{k}$ are defined by

$$
\mathbf{A}_{k}=\left(\begin{array}{ccccc}
A_{k}^{1,1} & A_{k}^{1,2} & 0 & \cdots & \cdots \\
A_{k}^{2,1} & A_{k}^{2,2} & A_{k}^{2,3} & \cdots & \cdots \\
& \ddots & \ddots & \ddots & \\
\cdots & \cdots & A_{k}^{M-1, M-2} & A_{k}^{M-1, M-1} & A_{k}^{M-1, M} \\
\cdots & \cdots & 0 & A_{k}^{M, M-1} & A_{k}^{M, M}
\end{array}\right)
$$

The other matrices $\mathbf{B}_{k}$ and $\mathbf{C}_{k}$ are also tridiagonal and defined similarly. The solution vectors $\Psi_{k-1}, \Psi_{k}, \boldsymbol{\psi}_{k+1}$ and the forcing vector $\mathbf{g}_{k}$ are defined as

$$
\boldsymbol{\Psi}_{k-1}=\left(\begin{array}{c}
\psi_{1, k-1} \\
\psi_{2, k-1} \\
\psi_{3, k-1} \\
\vdots \\
\psi_{M, k-1}
\end{array}\right), \quad \boldsymbol{\Psi}_{k}=\left(\begin{array}{c}
\psi_{1, k} \\
\psi_{2, k} \\
\psi_{3, k} \\
\vdots \\
\psi_{M, k}
\end{array}\right), \quad \boldsymbol{\Psi}_{k+1}=\left(\begin{array}{c}
\psi_{1, k+1} \\
\psi_{2, k+1} \\
\psi_{3, k+1} \\
\vdots \\
\psi_{M, k+1}
\end{array}\right), \quad \mathbf{g}_{k}=\left(\begin{array}{c}
g_{1, k} \\
g_{2, k} \\
g_{3, k} \\
\vdots \\
g_{M, k}
\end{array}\right)
$$

The components of the coefficient matrices are 


$$
\begin{array}{lll}
A_{k}^{j, j-1}=\frac{b_{j, k}}{4 \Delta y \Delta z}, & A_{k}^{j, j}=\frac{c_{j, k}}{\Delta z^{2}}-\frac{e_{j, k}}{2 \Delta z}, & A_{k}^{j, j+1}=-\frac{b_{j, k}}{4 \Delta y \Delta z} \\
B_{k}^{j, j-1}=\frac{a_{j, k}}{\Delta y^{2}}-\frac{d_{j, k}}{2 \Delta y}, & B_{k}^{, j}=f_{j, k}-\frac{2 a_{j, k}}{\Delta y^{2}}-\frac{2 c_{j, k}}{\Delta z^{2}}, & B_{k}^{j, j+1}=\frac{a_{j, k}}{\Delta y^{2}}+\frac{d_{j, k}}{2 \Delta y} \\
C_{k}^{j, j-1}=-\frac{b_{j, k}}{4 \Delta y \Delta z}, & C_{k}^{j, j}=\frac{c_{j, k}}{\Delta z^{2}}-\frac{e_{j, k}}{2 \Delta z}, & C_{k}^{j, j+1}=\frac{b_{j, k}}{4 \Delta y \Delta z}
\end{array}
$$

The bottom boundary condition (A2) is written in terms of the finite difference form for the interior points at the bottorn $(j=1, \cdots, M ; k=1)$,

$$
p_{j} \frac{\psi_{j+1,1}-\psi_{j-1,1}}{2 \Delta y}+q_{j} \frac{\psi_{j, 2}-\psi_{j, 1}}{\Delta z}+r_{j} \psi_{j, 1}=s_{j}
$$

and, by rearranging, as

$$
-\frac{p_{j}}{2 \Delta y} \psi_{j-1,1}+\left(r_{j}-\frac{q_{j}}{\Delta z}\right) \psi_{j, 1}+\frac{p_{j}}{2 \Delta y} \psi_{j+1,1}+\frac{q_{j}}{\Delta z} \psi_{j, 2}=s_{j}
$$

In matrix form, (A11) can be written as

$$
\mathbf{B}_{1} \Psi_{1}+\mathbf{C}_{1} \boldsymbol{\Psi}_{2}=\mathbf{g}_{1}
$$

where the coefficient matrix $\mathbf{B}_{1}$ is a tridiagonal matrix defined similarly to (A5) and $\mathbf{C}_{1}$ is a diagonal matrix defined by

$$
\mathbf{C}_{1}=\left(\begin{array}{ccccc}
C_{1}^{1,1} & 0 & 0 & \cdots & \cdots \\
0 & C_{1}^{2,2} & 0 & \cdots & \cdots \\
0 & 0 & C_{1}^{3,3} & \cdots & \cdots \\
& & & \ddots & \\
\cdots & \cdots & \cdots & \cdots & C_{1}^{M . M}
\end{array}\right)
$$

with the components

$$
B_{1}^{j, j-1}=-\frac{p_{j}}{2 \Delta y}, \quad B_{1}^{j, j}=r_{j, 1}-\frac{q_{j}}{\Delta z}, \quad B_{1}^{j, j+1}=\frac{p_{j}}{2 \Delta y}
$$




$$
C_{1}^{j, j}=\frac{q_{j}}{\Delta z}
$$

The solution vectors $\psi_{1}, \psi_{2}$ and the forcing vector $\mathbf{g}_{1}$ are defined as in (A8). The upper boundary condition (A3) is written in terms of the finite difference form for the interior points at the top $(j=1, \cdots, M ; k=N+1)$,

$$
t_{j} \frac{\psi_{j+1, N+1}-\psi_{j-1, N+1}}{2 \Delta y}+u_{j} \frac{\psi_{j, N+1}-\psi_{j, N}}{\Delta z}+v_{j} \psi_{j, N+1}=h_{j}
$$

and, by rearranging, as

$$
-\frac{u_{j}}{\Delta z} \psi_{j, N}-\frac{t_{j}}{2 \Delta y} \psi_{j-1, N+1}+\left(v_{j}+\frac{u_{j}}{\Delta z}\right) \psi_{j, N+1}+\frac{t_{j}}{2 \Delta y} \psi_{j+1, N+1}=h_{j}
$$

In matrix form, (A16) can be written as

$$
\mathbf{A}_{N+1} \boldsymbol{\Psi}_{N}+\mathbf{B}_{N+1} \boldsymbol{\Psi}_{N+1}=\mathbf{g}_{N+1}
$$

where the coefficient matrix $\mathbf{A}_{N_{+1}}$ is a diagonal matrix defined similarly to (A13) and $\mathbf{B}_{N+1}$ is a tridiagonal matrix defined similarly to (A7) with the components

$$
\begin{gathered}
A_{N+1}^{j, j}=-\frac{u_{j}}{\Delta z}, \\
B_{N+1}^{j, j-1}=-\frac{t_{j}}{2 \Delta y}, \quad B_{N+1}^{j, j}=v_{j}+\frac{u_{j}}{\Delta z}, \quad B_{N+1}^{j, j+1}=\frac{t_{j}}{2 \Delta y}
\end{gathered}
$$

The solution vectors $\boldsymbol{\Psi}_{N}, \boldsymbol{\Psi}_{N+1}$ and the forcing vector $\mathbf{g}_{N+1}$ are defined as in (A8).

The upper boundary condition depends on the physical property of $\psi$. As long as the top boundary condition can be written as in the (A3) form, the above formulation can be used. The examples are zero value, zero derivatives and radiation condition. 
The finite difference forms of the bottom boundary condition (A12) and top boundary condition (A17) yield another $2 \times M$ equations without extra unknowns. Hence the system is now closed to solve. We have a linear system with $M \times(N+1)$ equations and same number of unknowns. We follow the method proposed by Lindzen and Kuo (1969) as in Matsuno (1970). A modified version of this technique for the reduction of the memory was proposed by Schoeberl (1980) and used by Lin (1982).

The solution vector $\Psi_{k}$ is defined in a recursion relation as

$$
\boldsymbol{\Psi}_{k}=\mathbf{W}_{k} \boldsymbol{\Psi}_{k+1}+\boldsymbol{\delta}_{k}, \quad k=1, \cdots, N
$$

where $\mathbf{W}_{k}$ is a $(M \times M)$ matrix and $\boldsymbol{\delta}_{k}$ is a $(M \times 1)$ column vector. Substituting (A19) for $\psi_{k-1}$ into (A6) and rearranging the terms gives

$$
\boldsymbol{\Psi}_{k}=-\left(\mathbf{A}_{k} \mathbf{W}_{k-1}+\mathbf{B}_{k}\right)^{-1} \mathbf{C}_{k} \boldsymbol{\Psi}_{k+1}+\left(\mathbf{A}_{k} \mathbf{W}_{k-1}+\mathbf{B}_{k}\right)^{-1}\left(\mathbf{g}_{k}-\mathbf{A}_{k} \boldsymbol{\delta}_{k-1}\right)
$$

By comparison (A20) with (A19), we get relations for $\mathbf{W}_{k}$ and $\boldsymbol{\delta}_{k}$ as

$$
\begin{aligned}
& \mathbf{W}_{k}=-\left(\mathbf{A}_{k} \mathbf{W}_{k-1}+\mathbf{B}_{k}\right)^{-1} \mathbf{C}_{k} \\
& \boldsymbol{\delta}_{k}=\left(\mathbf{A}_{k} \mathbf{W}_{k-1}+\mathbf{B}_{k}\right)^{-1}\left(\mathbf{g}_{k}-\mathbf{A}_{k} \boldsymbol{\delta}_{k-1}\right)
\end{aligned}
$$

The lower boundary condition (A12) becomes

$$
\boldsymbol{\Psi}_{1}=-\mathbf{B}_{1}^{-1} \mathbf{C}_{1} \boldsymbol{\Psi}_{2}+\mathbf{B}_{1}^{-1} \mathbf{g}_{1}
$$

and the comparison between (A22) and (A19) gives

$$
\begin{aligned}
& \mathbf{W}_{1}=-\mathbf{B}_{1}^{-1} \mathbf{C}_{1} \\
& \boldsymbol{\delta}_{1}=\mathbf{B}_{1}^{-1} \mathbf{g}_{1}
\end{aligned}
$$

For the top boundary condition, substitution of (A19) for $\boldsymbol{\Psi}_{N}$ into (A17) and rearranging the terms gives 


$$
\boldsymbol{\Psi}_{N+1}=\left(\mathbf{A}_{N+1} \mathbf{W}_{N}+\mathbf{B}_{N+1}\right)^{-1}\left(\mathbf{g}_{N+1}-\mathbf{A}_{N+1} \boldsymbol{\delta}_{N}\right)
$$

The solution procedure is as follows:

(1) Obtain $\mathbf{B}_{1}, \mathbf{C}_{1}$ by (A14) and $\mathbf{g}_{1}$.

(2) Compute $\mathbf{W}_{1}$ and $\boldsymbol{\delta}_{1}$ by (A23) using $\mathbf{B}_{1}, \mathbf{C}_{1}$ and $\mathbf{g}_{1}$.

(3) Obtain $\mathbf{A}_{2}, \mathbf{B}_{2}$ and $\mathbf{C}_{2}$ by (A9) and $\mathbf{g}_{2}$.

(4) Compute $\mathbf{W}_{2}$ and $\boldsymbol{\delta}_{2}$ by (A21) using $\mathbf{A}_{2}, \mathbf{B}_{2}, \mathbf{C}_{2}, \mathbf{g}_{2}$ and $\mathbf{W}_{1}$ and $\boldsymbol{\delta}_{1}$

(5) Repeat the steps (3) and (4) to compute $\mathbf{W}_{k}$ and $\boldsymbol{\delta}_{k}$ for $k=3, \cdots, N$.

(6) Get $\boldsymbol{\Psi}_{N+1}$ by (A24).

(7) Solve for $\psi_{N}$ by (A19) using $\mathbf{W}_{N}, \delta_{N}$ and $\psi_{N+1}$.

(8) Repeat the step (7) to compute $\psi_{k}$ for $k=N-1, \cdots, 1$.

The above procedures are generally applicable to the equations of the form (A1) to (A3). In real calculations we usually have simpler top boundary condition. In those cases it is more convenient to use simpler step of (6) than using (A24). Two cases a. ? as follows:

1. Zero boundary

$$
\Psi_{N+1}=0
$$

2. Zero first derivative

Using $\boldsymbol{\psi}_{N+1}=\boldsymbol{\Psi}_{N}$ and $\boldsymbol{\Psi}_{N}=\mathbf{W}_{N} \boldsymbol{\Psi}_{N+1}+\boldsymbol{\delta}_{N}$ gives

$$
\boldsymbol{\Psi}_{N+1}=\left(\mathbf{I}-\mathbf{W}_{N}\right)^{-1} \boldsymbol{\delta}_{N}
$$




\section{REFERENCES}

Andrews, D. G., J. R. Holton, and C. B. Leovy, 1987: Middle atmosphere dynamics. Academic Press, New York, 489 pp.

Garcia, R. R., 1991: Parameterization of planetary wave breaking in the middle atmosphere. J. Atmos. Sci., 48, 1405-1419.

Holton, James R., 1975: The dynamic meteorology of the stratosphere and mesosphere. Meteorol. Monogr. No. 37, Amer. Meteorol. Soc., Boston, Mass.

Lin, B.-D., 1982: The behavior of winter stationary planetary waves forced by topography and diabatic heating. J. Atmos. Sci., 39, 1206-1226.

Lindzen, R. S., H.-L. Kuo, 1969: A reliable method for the numerical integration of a large class of ordinary and partial differential equations. Mon. Weather Rev., 97, 732-734.

Matsuno, T., 1970: Vertical propagation of stationary waves in the winter northern hemisphere. J. Atmos. Sci., 27, 871-883.

Schoeberl, M. R., 1980: Direct elliptic equation solvers with low memory requirements. NRL Memorandum Report 4191, Naval Research Lab., Washington, D. C., 18 pp. 

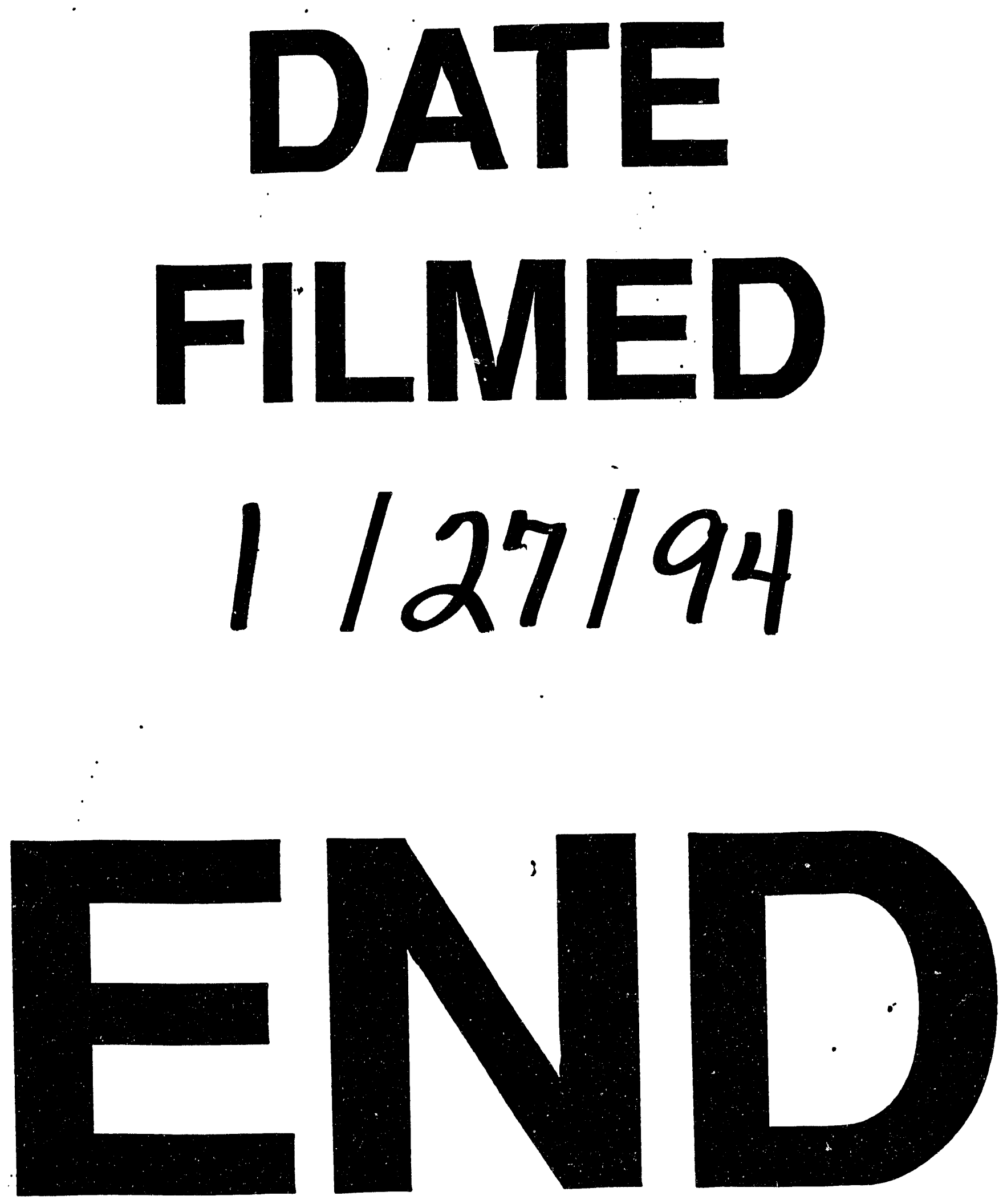
